\title{
Hemodilutional Autologous Blood Transfusion Reduces Postoperative 12h Chest Tube Drainage.
}

\author{
Hiroshi SAKAMOTO, Syunsuke EDAKUBO, Yoshihide MIURA, Katsumi HARASAWA \\ Department of Anesthesia, Hokkaido Ohno Memorial Hospital, Sapporo, 0630052, Japan
}

Background and Goal of Study: Blood transfusion in cardiac surgery with cardiopulmonary bypass (CPB) can correct anemia and coagulation abnormality. Hemodilutional autologous blood transfusion seems to have more advantages than allogeneic blood transfusion. We started hemodilutional autologous transfusion since April 2016. We compared hemodilutional autologous blood transfusion with allogeneic blood transfusion.

Patients and Methods: After obtaining IRB approval and informed consent, 235 patients undergoing scheduled cardiac surgery with CPB were enrolled in this study. They were consecutive 14 patients who received surgery with hemodilutional autologous blood transfusion (HAT group) and 221 patients whose surgery matches those patients (Control group). Anesthesia was induced and maintained with proporol, remifentanil, fentanyl and rocuronium. Blood salvage system was used in all cases. In HAT group, blood was collected after anesthesia induction and before skin incision from femoral venous line. The amount of blood to be removed was calculated with the following formula: $80 \times$ body weight $(\mathrm{kg}) \times(1-27 / \mathrm{Hct}(\%))$ with the utmost $800 \mathrm{ml}$. Removed blood was replaced with colloid solution. On site APTT, PT-INR and fibrinogen concentration (FIB) was measured with CG02N (A\&T Corporation, Yokohama, Japan). Those at the arrival in OR, heparin reversal and conclusion of surgery and blood loss during surgery, the amount of allogeneic blood transfusion and postoperative $12 \mathrm{~h}$ chest tube drainage were recorded. Data were compared using unpaired t-test. A $p$-value $<0.05$ was considered statistically significant.
Results and Discussion: Patients' demographic data, calculated amount of autologous blood collection and preoperative APTT, PT-INR and FIB showed no significant difference (Table 1.). At the arrival in OR, Hct in HAT group was significantly higher than that of Control group. At the heparin reversal, APTT, PT-INR showed no significant difference but FIB in HAT group was significantly lower than that of Control group. At the conclusion of surgery, they showed no significant difference again except significantly higher Hct in HAT group (Table 2). The amount of blood loss and allogeneic blood transfusion during surgery showed no significant difference (Table 3.). Twelve hours chest tube drainage in HAT group was significantly smaller than Control group (Table 3). In HAT group, hemodilution may result in lower FIB at the reversal of heparin. Calculated FIB which is the sum of FIB at heparin reversal and content of FIB in autologous blood per circulation volume is $182 \pm 53 \mathrm{mg} / \mathrm{dl}$ in HAT group and $165 \pm 63 \mathrm{mg} / \mathrm{dl}$ in Control group and they show no significant difference. Actually autologous blood transfusion may correct FIB at the conclusion of surgery. And autologous blood transfusion reduces postoperative $12 \mathrm{~h}$ chest tube drainage.

Conclusion: Hemodilutional autologous blood transfusion reduced postoperative $12 \mathrm{~h}$ chest tube drainage in cardiac surgery with $\mathrm{CPB}$.

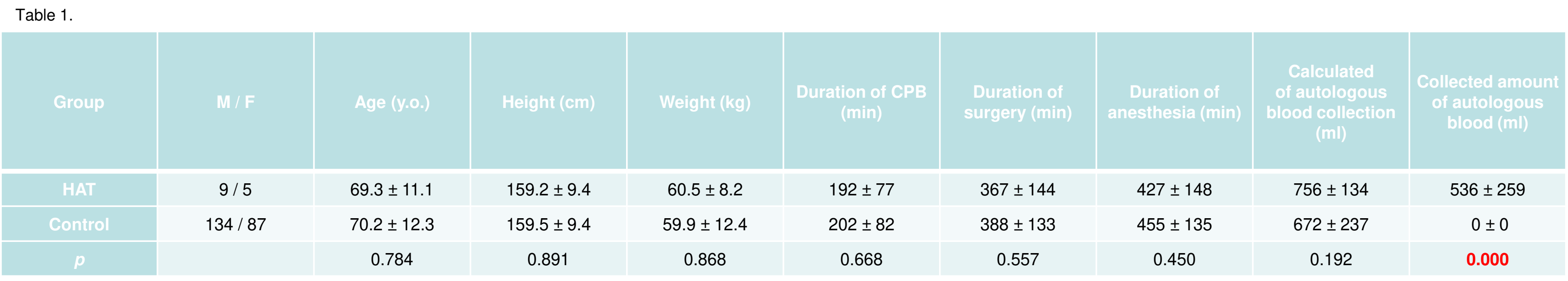

Data are shown in mean \pm S.D.

Table 2

\begin{tabular}{|c|c|c|c|c|c|c|}
\hline & Group & Het $(\%)$ & APTT (sec) & PT $(\%)$ & PT-INR & $\mathrm{F} \mid \mathrm{B}(\mathrm{mg} / \mathrm{d} \mid \mathrm{l})$ \\
\hline \multirow{3}{*}{$\begin{array}{l}\text { Arrival } \\
\text { in OR }\end{array}$} & HAT & $39.1 \pm 6.7$ & $27.5 \pm 4.2$ & $89.6 \pm 8.9$ & $1.08 \pm 0.08$ & $310 \pm 51$ \\
\hline & Control & $35.7 \pm 5.6$ & $31.2 \pm 8.8$ & $81.5 \pm 14.6$ & $1.19 \pm 0.23$ & $303 \pm 80$ \\
\hline & $p$ & 0.030 & 0.150 & 0.062 & 0.111 & 0.771 \\
\hline \multirow{3}{*}{ Heparin reversal } & HAT & $25.3 \pm 2.3$ & $44.9 \pm 8.4$ & $49.2 \pm 9.6$ & $1.85 \pm 0.56$ & $119 \pm 47$ \\
\hline & Control & $25.7 \pm 3.3$ & $55.4 \pm 3.3$ & $52.7 \pm 9.6$ & $1.71 \pm 0.42$ & $165 \pm 63$ \\
\hline & $p$ & 0.672 & 0.537 & 0.191 & 0.228 & 0.010 \\
\hline \multirow{3}{*}{$\begin{array}{l}\text { Conclusion } \\
\text { of surgery }\end{array}$} & HAT & $33.4 \pm 5.8$ & $34.8 \pm 8.1$ & $65.2 \pm 8.8$ & $1.39 \pm 0.19$ & $196 \pm 47$ \\
\hline & Control & $30.5 \pm 4.5$ & $37.9 \pm 8.6$ & $61.3 \pm 9.5$ & $1.47 \pm 0.21$ & $183 \pm 70$ \\
\hline & $p$ & 0.022 & 0.191 & 0.142 & 0.167 & 0.509 \\
\hline
\end{tabular}

Table 3.

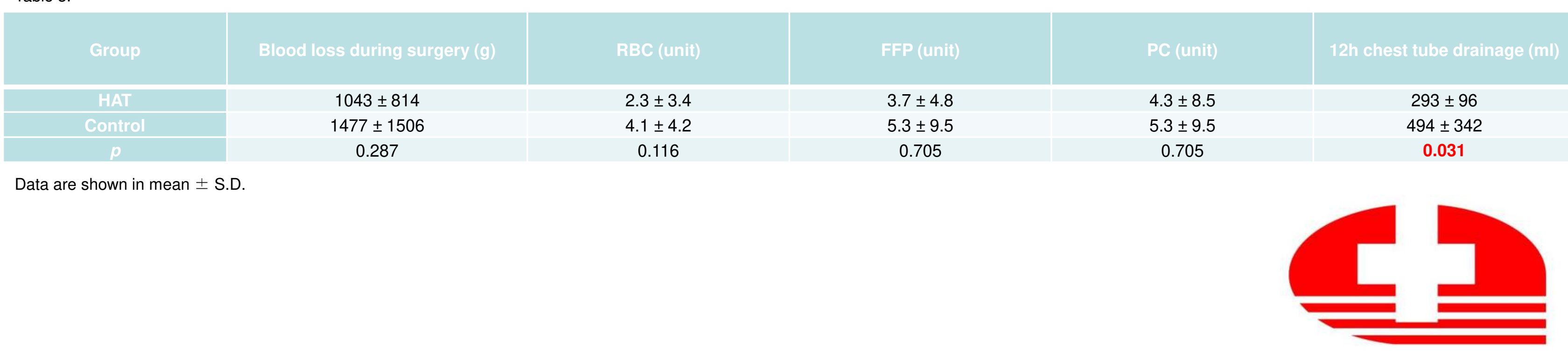

